\title{
Estructura valorativa de la motivación del alumnado de Formación Profesional hacia sus prácticas curriculares
}

\section{Valuation structure of the motivation of the students on Vocational Training towards their curricular practices}

Dra. M. Carmen Santos-González

Universidad de Santiago de Compostela

mcsantos@usc.es

Dra. M.Carmen Sarceda-Gorgoso

Universidad de Santiago de Compostela

carmen.sarceda@usc.es

Santos-González, M.C. \& Sarceda-Groso, M.C. (2017). Estructura valorativa de la motivación del alumnado de Formación Profesional hacia sus prácticas curriculares. Revista Prácticum, 2(1),22-39. 


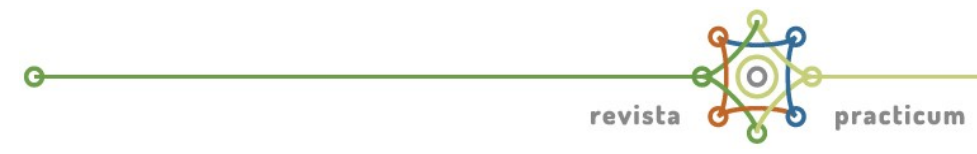

\section{Resumen}

El estudio en el que se enmarca este trabajo sitúa sus objetivos en una doble dimensión: conocer la valoración global de la motivación del alumnado con respecto a la realización de prácticas en la empresa, y establecer aquellos elementos que alcanzan una menor valoración para cada factor identificado. En el análisis estadístico de los datos empleamos técnicas descriptivas y técnicas multivariantes, concretamente el análisis de componentes principales para datos categóricos (CATPCA), a fin de obtener la información de las variables originales que configuran cada dimensión y ordenarlas según su valoración. Los resultados obtenidos muestran que el alumnado está altamente motivado, ya que globalmente el $77,5 \%$ de las puntuaciones son altas, frente al $9,1 \%$ que son bajas. Son los aspectos referidos a procesos relacionados directamente con las TIC y los recursos propios del ámbito laboral, así como otros relacionados con el desempeño del trabajo, los que alcanzan las valoraciones más bajas, lo que lleva a concluir la necesidad de intervenir en estos aspectos.

\section{Abstract}

The study which is part of this work puts its objectives in a double dimension: meet the overall assessment of the motivation of students with regard to practices in the company, and establish those elements that reach a lower rating for each factor identified. Analysis statistical data we employ descriptive and multivariate techniques, specifically the principal component analysis for categorical data (CATPCA), in order to obtain the information of the original variables that configure each dimension and order them according to their estimation. The results show that students are highly motivated, as globally $77.5 \%$ of the scores are high, compared with the $9.1 \%$ are low. They are the aspects relating to processes directly related to ICTs and equity in the workplace, as well as others related to the performance of the work, reaching the lower valuations, which leads to conclude the need to intervene in these aspects.

\section{Palabras claves}

Educación y formación profesional; periodo de prácticas profesionales; motivación; investigación educativa; trabajo.

\section{Keywords}

Vocational education and training; apprenticeship; motivation; educational research; labour. 


\section{Introducción}

En la actualidad la Formación Profesional (en adelante FP) está adquiriendo un peso importante a la hora de la elección de estudios por parte de los jóvenes que pretenden ampliar su formación, constatándose en la última década un incremento en el número de alumnos y alumnas que demandan este tipo de estudios. A su vez, desde los centros educativos son cada vez más las medidas que se ponen en marchar con la finalidad de ofertar una enseñanza de calidad, siguiendo la estela de las diferentes iniciativas llevadas a cabo en los distintos países del marco europeo con el objetivo de mejorar los sistemas de Formación Profesional (Lentzen, 2016).

Con la reforma realizada a partir de la LOGSE en 1990 se da un giro a una enseñanza demasiado academicista y alejada del mundo laboral (Sarceda-Gorgoso y Rial, 2011). Con esta Ley surge la obligatoriedad de la formación práctica a través de un periodo en la empresa que vincula el mundo educativo y el mundo laboral y que, desde el ámbito curricular se denomina Formación en Centros de Trabajo (en adelante FCT). A partir de este momento, se sigue reformulando el modelo adoptado en España, y actualmente se está apostando por darle un mayor peso a las prácticas, de ahí el nuevo modelo de FP Dual que se pone en marcha con el Real Decreto 1529/2012. Este modelo es reflejo de la tendencia actual en las formaciones profesionalizadoras -y más concretamente en la FP-, encaminada a incrementar el tiempo que ha de pasar el aprendiz en la empresa, lo cual le va a proporcionar una mejor adecuación el puesto de trabajo (Rego, Rial y Barreira, 2015). Sin embargo, estudios recientes (Marhuenda, Chisvert y Palomares, 2016) ponen de relieve determinadas debilidades del sistema, con valoraciones negativas por parte de los participantes y diferencias entre regiones, sectores profesionales y tipos de centros.

A pesar de esta situación de la FP Dual, la empresa se constituye en un escenario privilegiado para el desarrollo de competencias y así se reconoce desde el marco normativo, que regula la certificación y reconocimiento de la formación adquirida en contextos no formales y a través de la experiencia laboral, mediante la evaluación de competencias demostradas en el desempeño del trabajo directamente relacionadas con la formación. Esta situación no es exclusiva del contexto español sino que, tal y como señalan Marhuenda, Bernard y Navas (2010) es compartida en el ámbito europeo, donde se están desarrollando procedimientos de acreditación de los aprendizajes realizados más allá de las instituciones escolares.

En este proceso, el aprendiz ha de demostrar su conocimiento y su "saber hacer" en relación con las realizaciones profesionales, ya que serán los indicadores a emplear, y que como se afirma (Tejada, 2011) "cada día están tomando más relevancia en la evaluación de competencias, orquestándose verdaderas situaciones de simulación 


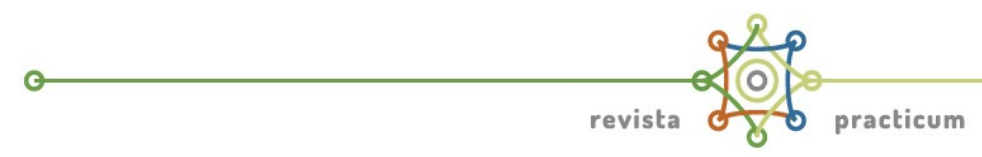

profesional." (p. 740). Así, el llegar a ser competente en una profesión requiere, tal y como señalan Álvarez, Asensio y García (2013) la integración de "elementos cognitivos y emocionales que lleven a la elección y puesta en marcha del protocolo de actuación más adecuado en cada caso y a un desempeño ético y de calidad, funcional, eficaz y eficiente" (p.143). Y en este desafío, las prácticas y la motivación del alumnado hacia ellas adquieren un papel fundamental de cara a promover procesos de calidad.

En la FP reglada, el módulo de la Formación en Centros de Trabajo es común a todos los ciclos formativos de Formación Profesional. Cada Título tiene un programa formativo específico que el alumnado desarrollará en una empresa perteneciente al sector productivo para el cual se está formando. Se realiza al finalizar el ciclo y como requisito para poder cursarlo ha de tener superados todos los módulos que integran dicho ciclo. La organización, el desarrollo y la ordenación de este módulo formativo, está regulado por el Real Decreto 1538/2006 donde se especifica que la finalidad de la FCT será:

- "Completar la adquisición de competencias profesionales propias de cada título alcanzadas en el centro educativo.

- Adquirir una identidad y madurez profesional motivadora para el aprendizaje a lo largo de la vida y para las adaptaciones a los cambios de las necesidades de cualificación.

- Completar conocimientos relacionados con la producción, la comercialización, la gestión económica y los sistemas de relaciones socio-laborales de las empresas, con el fin de facilitar la inserción laboral.

- Evaluar los aspectos más relevantes de la profesionalidad alcanzada por el alumnado en el centro educativo y acreditar los aspectos requeridos en el empleo que no puedan verificarse por exigir situaciones reales de trabajo" (p. 185).

En cuanto a su duración en los ciclos ya adaptados a LOE, y tal y como se establece en el currículo de cada ciclo, son 470 horas en las que el alumnado ha de adquirir los conocimientos y capacidades que se desarrollan en la empresa. Durante este periodo de prácticas, son dos los tutores los encargados de hacer un seguimiento del alumno/a que está realizando la FCT: el tutor del centro educativo y el tutor de la empresa. Ambos tutores han de estar en contacto a fin de que realmente el alumnado focalice su formación y realice aquellas tareas que le permitan adquirir las competencias requeridas en su titulación.

Decir además, que según el informe del Mercado de Trabajo Estatal 2014 (SEPE, 2015) "los trabajadores que están en posesión de títulos en Ciclos Formativos son especialmente valorados en el ámbito de la empresa y se encuentran entre los que tienen mejores posibilidades de inserción" (p. 4). Esta valoración por parte del mercado laboral, en buena medida viene determinada por las prácticas que los alumnos/as realizan 
en las empresas, siendo el número de horas bastante amplio y llevando un seguimiento por parte del centro educativo que permite determinar un plan individualizado para cada alumno, marcando unos objetivos concretos en su realización de tareas. En esta ejecución de las tareas, uno de los factores que van a incidir en su predisposición al abordaje de las mismas, va a ser la motivación con la que el alumnado afronta esta etapa en su formación y que pone en juego un amplio conjunto de procesos "cognitivos, afectivos, sociales y de carácter académico que se encuentran involucrados" (Steinmann, Bosch y Aisassa, 2013, pp. 586587) y en los que la comprensión y el análisis tienen una fuerte presencia (Soto y Torres, 2016).

Han sido muchos y muy diversos los campos en los que han sido estudiados los aspectos motivacionales referidos al proceso de enseñanza-aprendizaje (Steinmann et al., 2013; Soto y Torres, 2016). Sin embargo en nuestro caso, lo trasladamos al mundo del desarrollo académico-profesional, y cabe mencionar aquí el estudio llevado a cabo por Lozano y Repetto (2007) en el que da cuenta de un programa de desarrollo de la motivación, cuyo objetivo principal es promover un aprendizaje procedimental que incremente la capacidad de motivación que guíe los comportamientos, en este caso de cara a la realización de la FCT. Su fundamentación teórica la justifica considerando la motivación como una competencia de autorregulación intrapersonal, donde el propio alumno será el que la gestione durante el periodo de prácticas. Al mismo tiempo, se constituye en "una experiencia personal con gran capacidad de movilizar no solo intelectual sino también emocional y afectivamente a los estudiantes" (Zabalza, 2016). Otra aportación interesante la reallizan Maquilón y Hernández (2011), quienes en el estudio llevado a cabo con alumnado de formación profesional ponen de manifiesto que la motivación es el motor que mueve toda conducta, lo que permite provocar cambios tanto a nivel escolar como en la vida en general.

En el trabajo que presentamos, hemos tenido en consideración dicha competencia de autorregulación, formando parte de los principios en los que sustentamos nuestro estudio. Pero además consideramos la percepción del rol que van a desempeñar los alumnos/as, es decir, las actividades y comportamientos que serán precisos desarrollar para desempeñar eficazmente la tarea, así como el conocimiento de la organización del contexto de trabajo que, en consonancia con el esfuerzo que realice el alumnado (Porter y Lawler, 1968), serán el eje fundamental para su evolución durante las prácticas. Son estos los principios de las teorías procesuales de la motivación, en los cuales se enmarca y justifica nuestro trabajo.

Por otra parte, cabe señalar que los estudios centrados en la temática que aúna prácticas profesionales y motivación en las enseñanzas de formación profesional son muy escasos. Si bien es cierto que el prácticum y las prácticas profesionales han sido y son objeto de atención en la investigación educativa, tradicionalmente han centrado su 


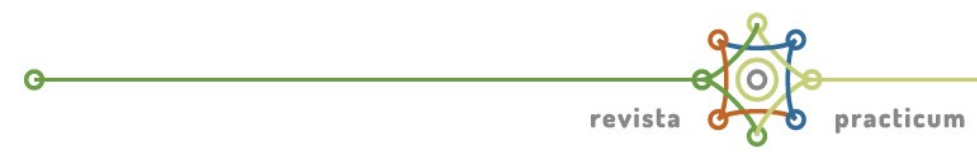

atención en la formación de maestros y, más recientemente, en el resto de formaciones universitarias. Con respecto a la formación práctica en FP, los esfuerzos se han dirigido fundamentalmente a evaluar el módulo de FCT (De la Fuente, 2001; Lorente, 2011; Marhuenda, 1994; Marhuenda et al., 2010; Rodríguez, 2002, 2005; Zurita, 2006), a tomarlo como eje para la definición de perfiles profesionales (Ruiz, 2008), o para vincular esta formación práctica con la inserción profesional (González, Cueto y Mato, 2006, 2008; Sarceda-Gorgoso y Rial, 2011).

De acuerdo con esto, resulta oportuno abordar la motivación de los alumnos y alumnas de Formación Profesional en relación con la Formación en Centros de Trabajo y que, vinculada a otras dimensiones ayudarán a explicitar los puntos clave a considerar en el diseño y desarrollo de este módulo de la formación.

El trabajo que se presenta se enmarca dentro de las actuaciones que se llevan a cabo en los centros públicos de Formación Profesional en la Comunidad Autónoma de Galicia. Más concretamente, se trata de un estudio realizado en un Centro Integrado de Formación Profesional perteneciente a la "Consellería de Cultura, Educación e Ordenación Universitaria" y que surge como iniciativa del propio centro -en el contexto de un proyecto de formación en centros- con la finalidad de conocer la motivación del alumnado respecto de la formación vinculada al módulo de FCT.

\section{Metodología}

\subsection{Objetivos}

Los objetivos que planteamos en este trabajo se sitúan en una doble dimensión:

- Conocer la valoración global de la motivación del alumnado con respecto a la realización de las prácticas en la empresa.

- Identificar el grado de motivación para cada uno de los aspectos planteados.

\subsection{Participantes}

Dado que el objetivo que perseguimos en el estudio es el de conocer la motivación del alumnado respecto al módulo de la FCT, nuestra unidad de análisis es dicho módulo formativo, que el alumnado va a cursar en el curso 2016/2017, siendo en este caso un total de 26 módulos, es decir, un módulo por ciclo formativo de los que están implantados en el CIFP. En nuestro caso la muestra está representada por un total de 14 módulos, lo que supone casi la mitad del total de 26 módulos, lo que consideramos que la muestra queda lo suficiente representada. 
Según la familia profesional mostramos en la Tabla 1 la distribución de los módulos que configuran la muestra, respecto al total de módulos existentes en los correspondientes ciclos formativos.

\begin{tabular}{cccccc}
\hline MÓDULOS FCT & $\begin{array}{c}\text { Frec.Centro } \\
\text { Módulo FCT }\end{array}$ & $\begin{array}{c}\text { \% Centro } \\
\text { Módulo FCT }\end{array}$ & $\begin{array}{c}\text { Frecuencia } \\
\text { Muestra }\end{array}$ & $\begin{array}{c}\% \text { Muestra } \\
\text { Módulo FCT }\end{array}$ & $\begin{array}{c}\text { Diferencia } \\
\text { (Sesgo) }\end{array}$ \\
\hline $\begin{array}{c}\text { FAMILIA } \\
\text { SANIDAD }\end{array}$ & 16 & $61,54 \%$ & 10 & $62,5 \%$ & $+0,97 \%$ \\
\hline $\begin{array}{c}\text { FAMILIA } \\
\text { SERVICIOS }\end{array}$ & 10 & $38,46 \%$ & 4 & $40 \%$ & $+1,54 \%$ \\
\hline TOTAL & 26 & $100 \%$ & 14 & $\begin{array}{c}100 \% \\
(53,84 \%)\end{array}$ & \\
\hline
\end{tabular}

Tabla 1, Distribución de los módulos de la FCT según la familia profesional, (Elaboración propia)

Al no existir una diferencia o sesgo importante, podemos considerar que la muestra de módulos es representativa para este centro.

\subsubsection{Características de los participantes}

Respecto a la distribución de la muestra según el sexo (Tabla 2), la gran mayoría son mujeres, el $80,9 \%$ frente al 19,1\% que son hombres; la mayoría tiene una edad situada entre los 18 y 25 años; y el $74,2 \%$ están cursando algún ciclo de la Familia Profesional de Sanitaria, frente al $25,8 \%$ de Servicios. En su mayor parte realizan un ciclo formativo de grado superior $(77,5 \%)$ y en régimen modular $(56,2 \%)$, siendo un $12,4 \%$ los ya tienen un título universitario y un $39,3 \%$ los que ya cursaron otro ciclo formativo.

\begin{tabular}{|c|c|c|c|}
\hline & & $\mathbf{n}$ & $\%$ \\
\hline \multirow{2}{*}{ Sexo } & Masculino & 17 & 19,1 \\
\hline & Femenino & 72 & 80,9 \\
\hline \multirow{6}{*}{ Edad } & Menos de 18 años & 1 & 1,1 \\
\hline & 18 a 20 años & 25 & 28,1 \\
\hline & 21 a 23 años & 25 & 28,1 \\
\hline & 24 a 26 años & 12 & 13,5 \\
\hline & 27 a 29 años & 6 & 6,7 \\
\hline & 30 o más años & 19 & 21,3 \\
\hline \multirow{2}{*}{ Familia Profesional } & Sanitaria & 66 & 74,2 \\
\hline & Servicios Socioc. y a la Comunidad & 23 & 25,8 \\
\hline \multirow{5}{*}{ Ciclo Formativo } & Atención a la dependencia & 3 & 3,4 \\
\hline & Integración Social & 20 & 22,5 \\
\hline & Audiología Protésica & 15 & 16,9 \\
\hline & Higiene Bucodental & 34 & 38,2 \\
\hline & Auxiliar de Enfermería & 17 & 19,1 \\
\hline \multirow{2}{*}{ Grado } & Medio & 20 & 22,5 \\
\hline & Superior & 69 & 77,5 \\
\hline \multirow{3}{*}{ Régimen } & Modular & 50 & 56,2 \\
\hline & Ordinario & 36 & 40,4 \\
\hline & Distancia & 3 & 3,4 \\
\hline \multirow{2}{*}{ Situación Laboral } & Trabajando & 25 & 28,1 \\
\hline & No trabaja & 64 & 71,9 \\
\hline Tiene otro ciclo de $\mathrm{F}$ & $\mathrm{Si}$ & 35 & 39,3 \\
\hline
\end{tabular}




\begin{tabular}{lllll} 
& & & \\
& & & & \\
\hline & revista & & 54 & 60,7 \\
\hline \multirow{2}{*}{ Título Universitario } & $\mathrm{Si}$ & 11 & 12,4 \\
\cline { 2 - 5 } & $\mathrm{No}$ & 78 & 87,6 \\
\hline
\end{tabular}

Tabla 2, Características del alumnado participante, (Elaboración propia)

\subsection{Instrumento y procedimiento}

En el trabajo de Santos-González y Sarceda-Gorgoso (2016) se da cuenta de las propiedades psicométricas de un cuestionario para conocer la motivación del alumnado de Formación Profesional, donde mediante la técnica del análisis factorial se comprobó que el instrumento elaborado denominado "Cuestionario para evaluar la Motivación del Alumnado hacia las Prácticas" (CUMAP), alcanza un índice de fiabilidad alto $(a=, 81)$.

Será éste el instrumento que utilizaremos para recoger la información, que tal y como es descrito por dichas autoras, está configurado por 16 variables, referidas a aspectos relacionados con la motivación y la planificación del trabajo, la motivación para el desempeño del mismo, la motivación cara a la inserción laboral, y la motivación por el aprendizaje y manejo de diferentes recursos.

En cuanto al procedimiento, los cuestionarios fueron aplicados en formato papel directamente y de forma intencional en el aula, durante el mes de junio del 2016 al alumnado de un Centro Integrado de Formación Profesional de las familias profesionales de Sanidad y Servicios Socioculturales y a la Comunidad, concretamente en la primera reunión que tienen con el tutor del centro educativo, en la cual se les facilita toda la información formal respecto al proceso de las prácticas en las empresas.

\subsection{Análisis de datos}

En el plan de análisis combinamos técnicas descriptivas y técnicas multivariantes: análisis de respuestas múltiples y CATPCA (componentes principales para datos categóricos). El tratamiento de los datos se llevó a cabo con el programa estadístico SPSS (Versión 22.0) para Windows.

\section{Resultados}

\subsection{Valoración global de la motivación}

Inicialmente interesa conocer la valoración global de la motivación del alumnado, para lo cual se analiza el conjunto de respuestas múltiples del conjunto de variables que configuran el instrumento. Agrupamos las respuestas correspondientes a las valoraciones de "Totalmente en desacuerdo" y "En desacuerdo" como único conjunto de repuestas, así como las valoraciones de "De acuerdo" y "Totalmente de acuerdo". Las puntuaciones asignadas a "Indiferente" son tratadas igualmente. Pretendemos, de esta forma, determinar si la valoración atribuida al grado de motivación es "Baja", "Media" o "Alta". 
Globalmente (Gráfico 1), se constata una elevada motivación para realizar las prácticas en las empresas $(77,5 \%$ de las puntuaciones otorgadas son altas y $9,1 \%$ a bajas).

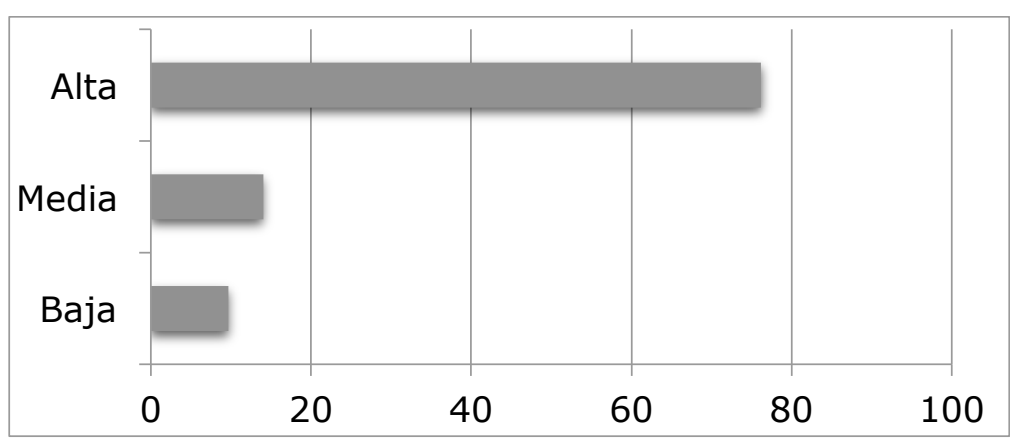

Gráfica 1. Distribución global de las puntuaciones para la motivación, (Elaboración propia)

Por otra parte, tal y como podemos comprobar en la Tabla 3, los estadísticos descriptivos las medias y rangos promedio son altos. La variable en la que se obtienen las puntuaciones más bajas son las referidas a "la preocupación o miedo al incorporarse a las prácticas" y, al "ritmo de trabajo en la empresa de prácticas".

\begin{tabular}{|c|c|c|c|c|}
\hline VARIABLES & $\mathbf{N}$ & Media & $\begin{array}{l}\text { Desviación } \\
\text { típica }\end{array}$ & $\begin{array}{c}\text { Rango } \\
\text { promedio }\end{array}$ \\
\hline $\begin{array}{l}\text { V01. La posible contratación por parte de la empresa } \\
\text { donde voy a realizar las prácticas. }\end{array}$ & 83 & 3,93 & 1,218 & 9,86 \\
\hline $\begin{array}{l}\text { V02. Lo que voy a aprender va a contribuir a } \\
\text { desarrollar las competencias del perfil profesional del } \\
\text { ciclo formativo. }\end{array}$ & 83 & 4,59 &, 606 & 10,15 \\
\hline $\begin{array}{l}\text { V03. Aprenderé sobre cómo organizar y planificar mi } \\
\text { trabajo. }\end{array}$ & 83 & 4,45 & 753 & 9,94 \\
\hline $\begin{array}{l}\text { V04. Encontrar un ambiente positivo en el centro de } \\
\text { prácticas. }\end{array}$ & 83 & 4,46 & ,650 & 10,83 \\
\hline $\begin{array}{l}\text { V05. Obtener méritos para posibles procesos } \\
\text { selectivos. }\end{array}$ & 83 & 4,14 & 1,037 & 10,90 \\
\hline $\begin{array}{l}\text { V06.El tener que pensar en distintas formas de cómo } \\
\text { desarrollar mi trabajo me ayudará encontrar trabajo } \\
\text { en el futuro. }\end{array}$ & 83 & 4,78 & 4,428 & 7,74 \\
\hline $\begin{array}{l}\text { V07. El planificar las actividades que tengo que llevar } \\
\text { a cabo me ayudará a ser más metódico y más } \\
\text { organizado/a. }\end{array}$ & 83 & 4,40 & ,764 & 9,55 \\
\hline $\begin{array}{l}\text { V08. Aprender a utilizar las TICaplicadas en el } \\
\text { ámbito laboral para el que me estoy preparando.. }\end{array}$ & 83 & 3,87 & 1,145 & 9,20 \\
\hline $\begin{array}{l}\text { V09. Conocer los recursos necesarios para } \\
\text { desarrollar mi trabajo. }\end{array}$ & 83 & 4,42 &, 646 & 9,45 \\
\hline V10. Útiles para mi capacitación profesional. & 83 & 4,59 &, 585 & 7,23 \\
\hline $\begin{array}{l}\text { V11. Me preocupa el ritmo de trabajo que tenga que } \\
\text { realizar. }\end{array}$ & 82 & 3,13 & 1,312 & 3,59 \\
\hline V12. Me da miedo incorporarme a la FCT. & 83 & 2,46 & 1,425 & 5,36 \\
\hline $\begin{array}{l}\text { V13.Me van a permitir el desarrollo de destrezas y } \\
\text { habilidades necesarias en el ámbito laboral. }\end{array}$ & 83 & 4,27 & ,734 & 6,14 \\
\hline $\begin{array}{l}\text { V14. La realización de la FCT podrá aumentar mi } \\
\text { motivación en el trabajo para el que me capacitará } \\
\text { mi titulación. }\end{array}$ & 65 & 4,34 & 834 & 8,04 \\
\hline $\begin{array}{l}\text { V15. Es conveniente que la asignación del alumnado } \\
\text { a la empresa, esté en función de intereses similares }\end{array}$ & 64 & 3,55 & 1,007 & 8,85 \\
\hline
\end{tabular}


entre ambas partes para poder compartir

experiencias profesionales.

V16. El número de horas que voy a realizar en la FCT

considero que son las adecuadas para conocer el

$65 \quad 3,88$

1,083

9,17

ámbito laboral del ciclo que estoy cursando.

Tabla 3. Estadísticos descriptivos de las variables, (Elaboración propia)

\subsection{Estructura valorativa}

Una vez que comprobamos la valoración global de la motivación del alumnado, nos interesa conocer cómo se estructura la valoración de la misma. Inicialmente, a fin de reducir la dimensionalidad de los datos, aplicamos el análisis de componentes principales para datos categóricos (CATPCA).

\begin{tabular}{ccc}
\hline Dimensión & $\begin{array}{c}\text { Alfa de } \\
\text { Cronbach }\end{array}$ & $\begin{array}{c}\text { Total } \\
\text { (Autovalores) }\end{array}$ \\
\hline 1 &, 911 & 6,854 \\
\hline 2 &, 517 & 1,941 \\
\hline Total &, 945 & 8,794 \\
\hline Tabla 4. Resumen del modelo, (Elaboración propia)
\end{tabular}

En la reducción del conjunto de variables obtenemos de forma intencional 2 dimensiones, para lo que empleamos el método de normalización principal por variable, ya que optimiza la asociación entre las variables, obteniendo así la saturación en la dimensión y en los componentes (Tabla 5).

\begin{tabular}{c|cc}
\hline \multirow{2}{*}{ Variables } & \multicolumn{2}{c}{ Dimensión } \\
\cline { 2 - 3 } & 1 & \multicolumn{1}{c}{2} \\
\hline V01 &, 475 &, 036 \\
\hline V02 &, 838 &, 048 \\
\hline V03 &, 758 &, 074 \\
\hline V04 &, 753 &,- 069 \\
\hline V05 &, 659 &, 071 \\
\hline V06 &, 695 &, 173 \\
\hline V07 &, 722 &, 420 \\
\hline V08 &,- 359 &, 683 \\
\hline V09 &, 895 &,- 074 \\
\hline V10 &, 849 &, 030 \\
\hline V11 &,- 269 &, 740 \\
\hline V12 &,- 454 &, 604 \\
\hline V13 &, 727 &, 040 \\
\hline V14 &, 664 &, 199 \\
\hline V15 &, 346 &, 503 \\
\hline V16 &, 572 &,- 188 \\
\hline
\end{tabular}

Tabla 5. Saturaciones en componentes, (Elaboración propia)

Normalización principal por variable

Nuestro interés se centra en saber cómo se agrupan las valoraciones en los distintos componentes a fin de poder así reducir la 
información y conocer el grado de valoración para la motivación, en cada uno de ellos (Imagen 1).

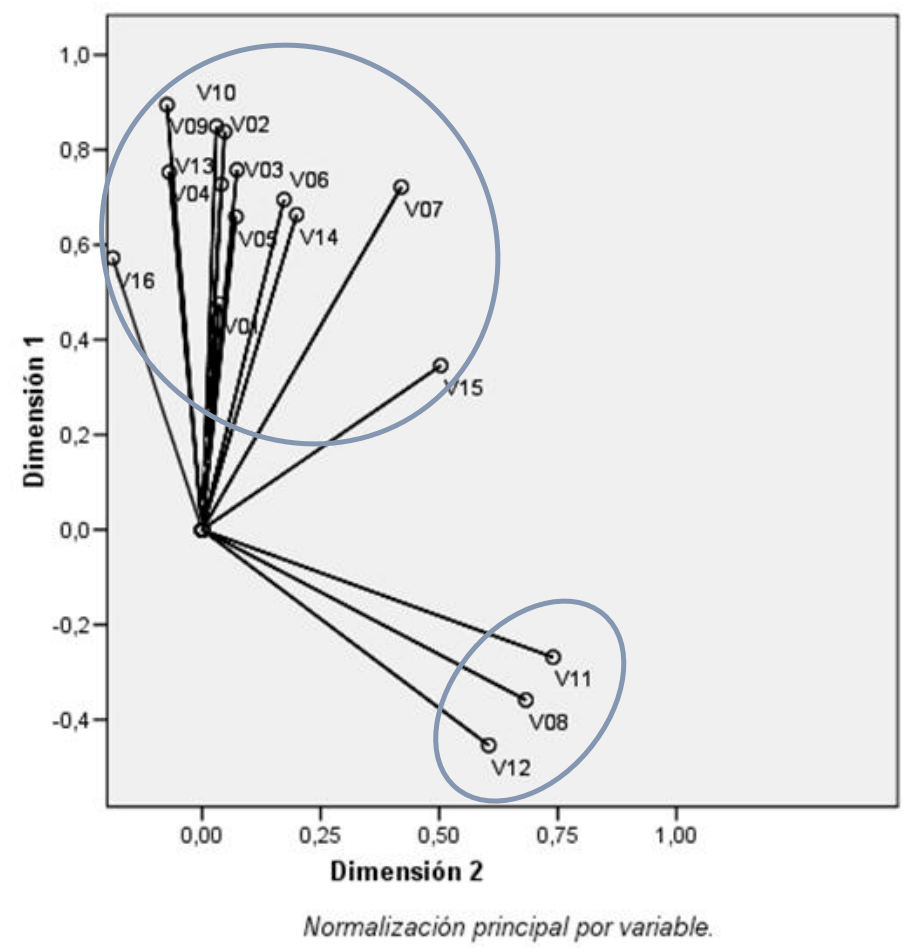

Ilustración 1. Saturaciones en los componentes para la motivación, (Elaboración propia)

Claramente, el alumnado configura la estructura de la valoración de su motivación en los dos grupos o factores, agrupando en la primera dimensión (polo + ) aquellos aspectos por los que se siente motivado relacionados con el desarrollo del trabajo y su percepción sobre su futura inserción laboral. Hemos denominado a esta dimensión "Trabajo e inserción laboral".

El segundo grupo de variables (polo -) tan sólo agrupa aquellos aspectos relacionados con las TIC y el ritmo de trabajo que ha de realizar, si bien cabe mencionar que es en esta dimensión donde sitúa también su miedo ante la incorporación a la empresa. Hemos denominado a esta dimensión "TIC y ritmo de trabajo".

En el Gráfico 2 mostramos como se ordenan los elementos de la motivación en cada factor según las puntuaciones de orden (rango) alcanzadas en su conjunto. 

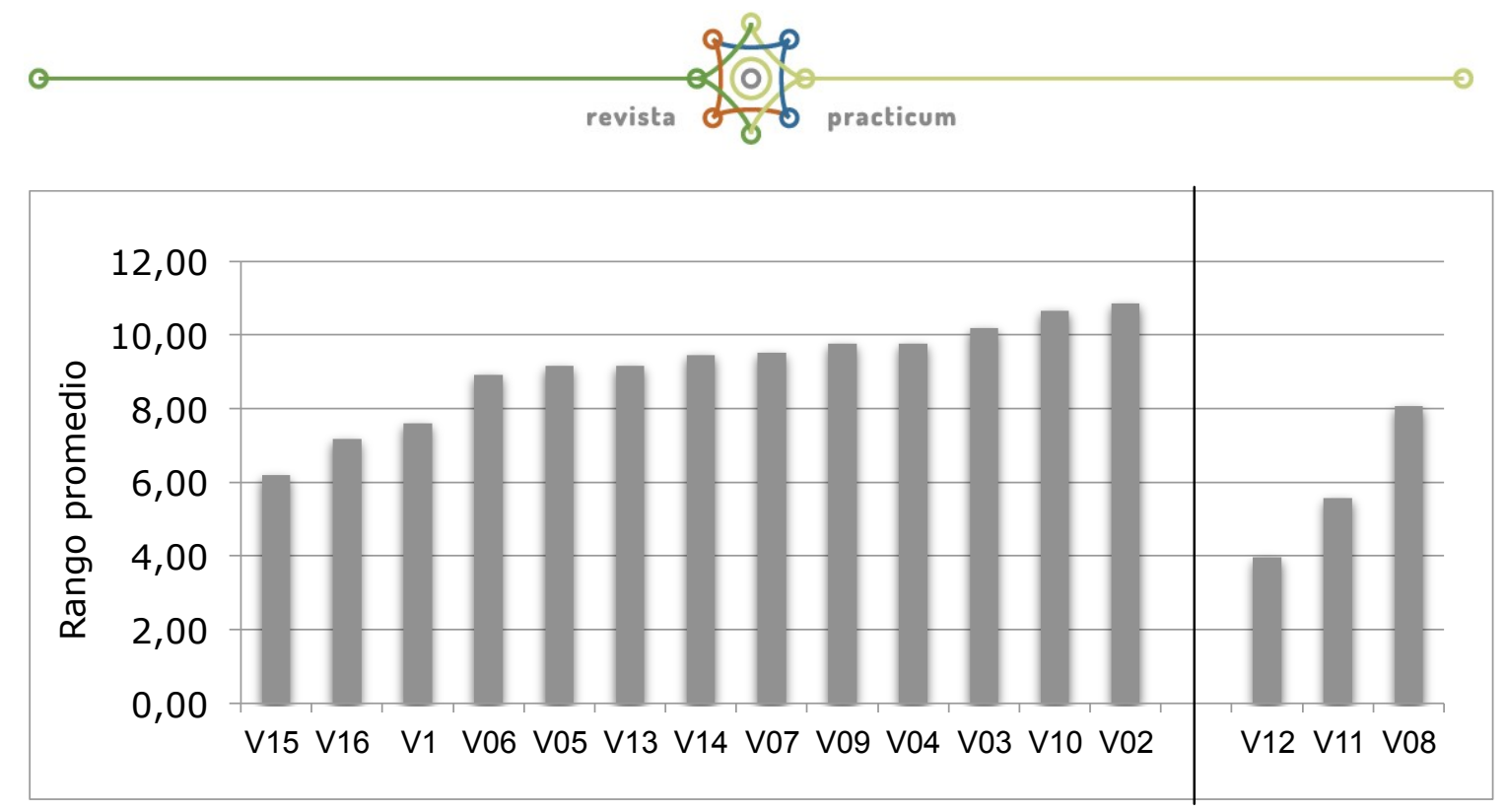

Gráfica 2. Ordenación de las variables según valoración para cada componente,

(Elaboración propia)

El grupo de variables que configuran el factor "Trabajo e inserción laboral" son las que alcanzan la mayor valoración promedio en las respuestas respecto a la motivación. Aquéllas referidas a la posibilidad de desarrollar las competencias propias del perfil profesional, la utilidad de las prácticas en la empresa para la capacitación profesional, y aprender cómo organizar y planificar el trabajo, alcanzan las mayores puntuaciones en la valoración.

El desarrollo de competencias del perfil profesional del ciclo formativo, y su utilidad para la capacitación profesional, representan el $65 \%$ de la valoración asignada por el alumnado el que se posiciona en "totalmente de acuerdo" frente al 6\% que se manifiesta indiferente, no siendo ninguno el que indica "totalmente en desacuerdo".

Otro de los aspectos que alcanza valores altos en la puntuación es el referido al ambiente positivo que esperan encontrar en el centro de prácticas, así como aprender a organizar y planificar su trabajo y conocer los recursos necesarios para ello, aspectos todos ellos directamente relacionados con el puesto de trabajo a desempeñar. Un $58 \%$ responde "totalmente de acuerdo", frente al $6 \%$ que se muestra indiferente, no siendo ninguno el que atribuye una valoración en "totalmente desacuerdo".

Con relación a aquéllos a los que el alumnado atribuye valoraciones menores -aunque mayoritariamente se posicionan con valores intermedios- destacan los referidos a la posible contratación por parte de la empresa en la que realizan las prácticas. Así, el $42 \%$ indica que está "totalmente de acuerdo" respecto al grado de motivación referida a este aspecto, frente al $20 \%$ que atribuye una valoración baja, y posicionándose los restantes en una posición intermedia. 


\section{Conclusiones y discusión}

De los resultados expuestos, lo primero que hemos de señalar es que globalmente, el alumnado de Formación Profesional indica que está motivado para emprender su fase de prácticas en las distintas empresas, encontrándose valores altos en la mayoría de los aspectos que fueron sometidos a valoración. Sin embargo hay otros aspectos sobre los que los alumnos y alumnas se posicionan en una situación intermedia, lo que nos lleva a deducir que si desde el centro desean conocer los aspectos en los cuales han de incidir en la información, son precisamente las posiciones más bajas e intermedias las que van a ser la guía para el diseño de la intervención.

El alumnado diferencia claramente los aspectos referidos al desempeño del trabajo y la inserción laboral, de los recursos propios del ámbito laboral y las posibles inquietudes ante su incorporación a la empresa de prácticas. Es precisamente en esta dimensión que hemos denominado "Inquietudes y TIC", donde las puntuaciones obtenidas son las menores.

Tal y como hemos podido comprobar, las TIC y el conocimiento de los recursos propios de su trabajo, son los aspectos que alcanzan una menor valoración. Creemos que este aspecto debe ser analizado con profundidad desde el centro educativo, tanto por el profesor tutor de la FCT como por el resto de profesorado de cada ciclo. Es ahí donde precisamente se inician en esta formación, que no debería centrarse sólo en las TIC como medio para el desarrollo de los conocimientos teóricos de los distintos módulos formativos (no debemos olvidar que el alumnado pertenece a una generación con altas competencias a nivel tecnológico desarrollas en el marco de la educación informal), sino avanzar hacia las que se vienen denominando TAC (Tecnologías del Aprendizaje y el Conocimiento) como medio para la realización de otros aprendizajes relacionados con el perfil profesional del título y que serán propios de su ámbito laboral. Esta idea nos lleva a pensar que, actualmente, el alumnado de los ciclos formativos utiliza las TIC pero no como Tecnologías para el Aprendizaje y el Conocimiento, para lo que tendrían que cumplir con la condición de facilitar el aprendizaje de conceptos, procedimientos y valores (Pariente y Perochena, 2013).

Respecto de la dimensión que denominamos "Trabajo e inserción laboral", por los resultados obtenidos en los análisis de los datos, consideramos de gran relevancia que el alumnado tenga conocimiento de la empresa previamente a su incorporación. En este sentido resultaría de gran interés un acercamiento de la empresa al centro previamente a su incorporación a la misma. La intervención que se diseñe al respecto, ha de contemplar la posibilidad de que los responsables y tutores de empresa, ya bien mediante charlas, reuniones con alumnos, jornadas, etc..., se acerquen al centro educativo, donde se clarificarán los puestos de trabajo y las actividades que se realizan en dichas empresas, aspecto 


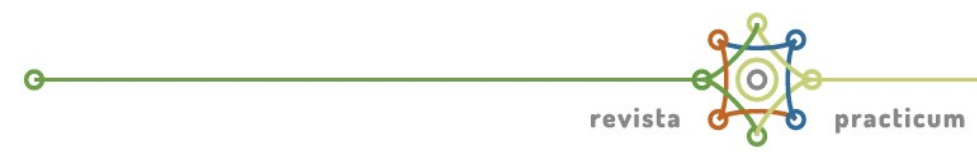

que se pone de manifiesto en otros estudios sobre las prácticas en el ámbito universitario (Caso y Alonso-Cortés, 2016) o más concretamente sobre la FCT (Marhuenda, 1994; Zurita, 2006; Lorente, 2011). Al mismo tiempo posibilitaría a los profesores de los centros educativos una forma excelente de mantener actualizado su conocimiento de los entornos laborales (González et al., 2006, 2008).

Por otra parte, y en el marco de las acciones anteriormente mencionadas, se informará al alumnado de la importancia de la adaptación al puesto de trabajo, para lo cual los tutores de empresa serían una fuente importante a la hora de tratar este aspecto, como ya se pudo de manifiesto en otros estudios precedentes (Sarceda y Rial, 2011).

Centrándonos en la inserción laboral, cabe mencionar el estudio llevado a cabo por González et al. (2006), en el cual concluyen que "la colaboración que se establece entre centros educativos y empresas tiene importantes beneficios para todas las partes implicadas" (p. 56), ya que el estudiante inicia su contacto con el mundo de la empresa que muchas veces da lugar a un posterior contrato de trabajo. En algunas empresas se decantan por adoptar políticas concretas acerca de la relación existente entre la formación y la inserción, existiendo la posibilidad de insertarse dentro de las mismas tras el período de prácticas, de modo que las utilizan como mecanismo de selección de personal, a corto o a largo plazo (Martínez, 2000). Esta dimensión era una de las que más motivación provocaba en el alumnado y vemos como se corrobora con los datos aportados por otros estudios que demuestran que la inserción laboral en la propia empresa de la FCT se situaba, en el conjunto de las familias profesionales, en un $17 \%$ un año después de haber finalizado los estudios (Sarceda y Rial, 2011), y específicamente en las familias de Sanidad y Servicios Socioculturales y a la Comunidad -en las centramos nuestra atención- en el $14 \%$ y $8 \%$, respectivamente (Gobierno de Cantabria, 2015).

Por este motivo, desde el centro educativo se le ha de proporcionar información sobre el ámbito laboral del ciclo formativo que están cursando. Es labor del Departamento de Información y Orientación el realizar este proceso, siendo recomendable que se profundice más en ello. Esta información deberá realizarse previamente a la realización de las prácticas, ya que desde la empresa podrán visibilizar aquellos aspectos y competencias que han de poseer, y sobre todo aquellas habilidades y métodos propios que requieren los puestos de trabajo propios de su titulación, así como las características propias del ámbito y las empresas objeto de contratación.

Como ya señalamos, estas expectativas y motivaciones son previas a la realización de la FCT, por lo que resulta necesario contrastarlas con la percepción posterior, abriéndose nuevas líneas de investigación. También sería importante contar con la visión de los otros participantes (tutores del centro y de la empresa), de manera que se puedan definir 
fortalezas y debilidades de la Formación en Centros de Trabajo que contribuyan a una optimización de este módulo de formación.

\section{Referencias}

Álvarez, M.M., Asensio, I. y García, J.M. (2013). Deporte y competencias genéricas en la universidad: diseño y validación del "competest". Revista Complutense de Educación, 24(1), 141-163. Recuperado de https://goo.gl/ywLSXu

Caso, A. y Alonso-Cortés, M.D. (2016). Procedimiento y Evaluación del Prácticum en la Facultad de Educación de la Universidad de León. Revista Prácticum, 1(1), 114-122. Recuperado de https://goo.gl/Oi8opr

De la Fuente, R. (2001). La Formación Profesional: formación en centros educativos y formación en centros de trabajo. Necesidades y demandas en Burgos. Tesis doctoral. Burgos: Universidad de Burgos.

Gobierno de Cantabria (2015). Informe de inserción laboral 2013-2014. Santander: Consejería de Educación, Cultura y Deporte. https://goo.gl/XAQ1N2

González, C.; Cueto, B. y Mato, F.J. (2006). El papel de la FCT en la inserción laboral de los titulados de ciclos formativos: el caso de Asturias. Revista de Educación, 341, 337-372.

González, C.; Cueto, B. y Mato, F.J. (2008). ¿Qué beneficios tiene para la empresa su colaboración con el sector educativo? Un análisis de la Formación en Centros de Trabajo desde la perspectiva empresarial. Revista del Ministerio de Trabajo e Inmigración, 76, 39-58.

Lentzen, S. (2016). Los modelos de formación dual como integración de lo académico y lo laboral. Revista Prácticum, 1(1), 24-39. https://goo.gl/kwiqTV

Lorente, R. (2011). La reforma de la Formación Profesional en España a través del diseño por competencias: un análisis desde la perspectiva de los agentes sociales. Tesis Doctoral. Granada: Universidad de Granada.

Lozano, S. y Repeto, E. (2007). Motivación y desarrollo profesional: un estudio piloto. Revista Electrónica de Motivación y Emoción, 10(25). https://goo.gl/QZbuj8 


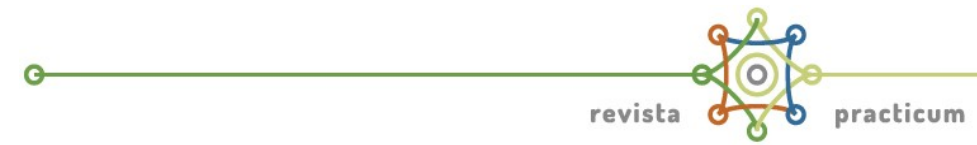

Maquilón, J.J. y Hernández, F. (2011). Influencia de la motivación en el rendimiento académico de los estudiantes de formación profesional. Revista Electrónica Interuniversitaria de Formación del Profesorado, 14(1),81-100. https://goo.gl/Gos57k

Marhuenda, F. (1994). Estudio y trabajo: la alternancia en la Formación Profesional. Tesis doctoral. Valencia: Universidad de Valencia.

Marhuenda, F., Chisvert, M.J. y Palomares, D. (2016). La formación profesional dual en España: Consideraciones sobre los centros que la implementan. RIO: Revista Internacional de Organizaciones, 17, 43-63. https://goo.gl/okhiBB

Marhuenda, F.; Bernard, J.C. y Navas, A. (2010). Las prácticas en empresa como estrategia de enseñanza e inserción laboral: las empresas de inserción social. Revista de Educación, 351, 139-161.

Martínez, M.J. (2000). Reflexiones acerca de la alternancia: una comparación entre el modelo de prácticas a través del módulo FCT (Formación en Centros de Trabajo) y el aprendizaje en España. Contextos Educativos, 3, 297-314. https://goo.gl/mh1Msk

Pariente, J.L. y Perochena, P. (2013). Didáctica de la educación en valores en la eso. Una propuesta utilizando las tecnologías para el aprendizaje y el conocimiento. Pixel-Bit: Revista de medios y educación, 42, 195-208. https://goo.gl/HM6f8I

Porter, L.W. y Lawler, E.E. (1968). What job attitudes can tell us about employee motivation. Harvard Business Review, 46(1), 118-126.

Real Decreto 1529/2012, de 8 de noviembre, por el que se desarrolla el contrato para la formación y aprendizaje y se establecen las bases de la formación profesional dual (BOE núm. 270, de 9 de noviembre de 2012).

Real Decreto 1538/2006, de 15 de diciembre, por el que se establece la ordenación general de la formación profesional del sistema educativo (BOE núm. 3, de 3 de enero de 2007).

Rego, L.; Rial, A. y Barreira, E.M. (2015). La formación en alternancia en la universidad y en los ciclos formativos: aportaciones desde dos investigaciones realizadas en Galicia. Educar, 51(2), 349-371. DOI: http://dx.doi.org/10.5565/rev/educar.687.

Rodríguez, M. (2002). Análisis del módulo de formación en centros de trabajo en la familia profesional administrativa en la Comunidad de Madrid. Enfoque desde la Pedagogía Laboral. Tesis Doctoral. Madrid: Universidad Complutense de Madrid. 
Rodríguez, M. (2005). La Formación en Centros de Trabajo: datos sobre su desarrollo en la familia Administración en la Comunidad de Madrid. Revista Complutense de Educación, 16(1), 255-288. https://goo.gl/i2seBU

Ruíz, J. (2008). Análisis del perfil profesional del "técnico superior en animación de actividades físicas y deportivas" a través de la "Formación en Centros de Trabajo". Tesis doctoral. Las Palmas: Universidad de Las Palmas de Gran Canaria.

Santos-González, M.C. y Sarceda-Gorgoso, M-C. (2016). La motivación del alumnado de Formación Profesional con respecto a sus prácticas profesionales: validación de un instrumento. En Rial, A.; Valcarce, M. y Rego, L., A formación, a orientación e o emprego no recoñecemento, avaliación e certificación de competencias profesionais adquiridas en contextos formais, non formais e informais (pp. 209-223). Santiago de Compostela: Campus na nube.

Sarceda-Gorgoso, M-C. y Rial, A. (2011). De las prácticas en alternancia a la inserción laboral: resultados de una investigación. Revista de Docencia Universitaria (REDU), 9(2), 231-252. https://goo.gl/NT96VC

SEPE (2015). Informe del Mercado de Trabajo Estatal. Datos 2014. Madrid: Servicio Público de Empleo Estatal. Recuperado de https://goo.gl/tg08xy

Soto, J.L. y Torres, C.A. (2016). Percepciones y expectativas del aprendizaje en jóvenes universitarios. REDU. Revista de Docencia Universitaria, 14(1), 51-67. DOI: http://dx.doi.org/10.4995/redu.2016.5797

Steinmann, A., Bosch, B. y Aiassa, D. (2013). Motivación y expectativas de los estudiantes por aprender ciencias en la universidad. Revista Mexicana de Investigación Educativa. 18(57), 585-598. https://goo.gl/QKa8Ag

Tejada, J. (2011). La evaluación de las competencias en contextos no formales: dispositivos e instrumentos de evaluación. Revista de Educación, 354, 731-745. http://dx.doi.org/10.4438/1988-592X-RE2011-354-018.

Zabalza, M.A. (2016). El Practicum y las prácticas externas en la formación universitaria. Revista Prácticum, 1(1), 1-23. https://goo.gl/JilqUk

Zurita, F.J. (2006). Análisis descriptivo de la formación en centros de trabajo (FCT) de familias profesionales técnico-industriales en el ámbito 


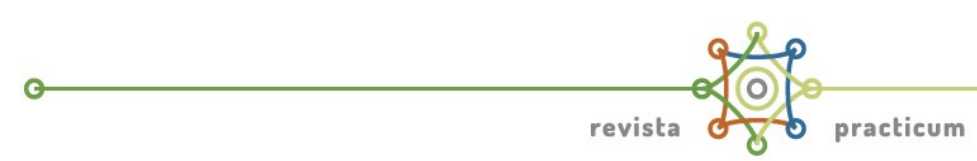

provincial de Zaragoza. Tesis Doctoral. Tarragona: Universidad Rovira i Virgili. 